\title{
Dissipative soliton resonance in a full polarization-maintaining fiber ring laser at different values of dispersion
}

\author{
I. Armas-Rivera, ${ }^{1,2}$ C. Cuadrado-Laborde,${ }^{1,3}$ A. Carrascosa, ${ }^{1}$ E. A. Kuzin,,${ }^{4, *}$ G. Beltrán- \\ Pérez, ${ }^{2}$ A. Díez, ${ }^{1}$ and M. V. Andrés ${ }^{1}$ \\ ${ }^{1}$ Departamento de Física Aplicada, ICMUV, Universidad de Valencia, Dr. Moliner 50, Burjassot E-46100, Spain \\ ${ }^{2}$ Benemérita Universidad Autónoma de Puebla, Facultad de Ciencias Físico Matemáticas Avenida San Claudio y 18 \\ Sur, Col. San Manuel CU Puebla 72570, Mexico \\ ${ }^{3}$ Instituto de Física Rosario (CONICET-UNR), Blvr. 27 de Febrero 210bis, S2000EZP, Rosario, Argentina \\ ${ }^{4}$ INAOE Enrique Erro 1, Tonanzintla, Puebla, Pue. 72000, Mexico \\ "ekuz@inaoep.mx
}

\begin{abstract}
We investigated the dissipative solitons resonance in an ytterbium-doped fiber ring laser in which all the elements are polarization maintaining (PM). A semiconductor saturable absorber mirror was used as a mode-locker. The cavity included a normal dispersion single-mode fiber (SMF) and an anomalous dispersion photonic crystal fiber. The change of the length of the PM SMF allows the variation of the net-normal dispersion of the cavity in the range from $0.022 \mathrm{ps}^{2}$ to $0.262 \mathrm{ps}^{2}$. As the absolute value of the net-normal dispersion increases from $0.022 \mathrm{ps}^{2}$ to $0.21 \mathrm{ps}^{2}$, a squareshaped single pulse transformed to a single right-angle trapezoid-shaped pulse, and, at the dispersion of $0.262 \mathrm{ps}^{2}$, to multiple right-angle trapezoidshaped pulses, per round-trip.
\end{abstract}

(C) 2016 Optical Society of America

OCIS codes: (060.4370) Nonlinear optics, fibers; (140.3510) Lasers, fiber; (060.5530) Pulse propagation and temporal solitons.

\section{References and links}

1. I. N. Duling, "All-fiber ring soliton laser mode locked with a nonlinear mirror," Opt. Lett. 16(8), 539-541 (1991).

2. D. J. Richardson, R. I. Laming, D. N. Payne, M. W. Phillips, and V. J. Matsas, " 320 fs soliton generation with passively mode-locked Erbium-fiber laser," Electron. Lett. 27(9), 730-732 (1991).

3. V. I. Matsas, T. P. Newson, D. J. Richardson, and D. N. Payne, "Self-starting passively mode-locked fiber ring soliton laser exploiting nonlinear polarization rotation," Electron. Lett. 28(15), 1391-1393 (1992).

4. V. I. Matsas, T. P. Newson, and M. N. Zervas, "Self-starting passively mode-locked fiber ring laser exploiting nonlinear polarization switching," Opt. Commun. 92(1-3), 61-66 (1992).

5. N. Akhmediev, J. M. Soto-Crespo, and Ph. Grelu, "Roadmap to ultra-short record high-energy pulses out of laser oscillators," Phys. Lett. A 372(17), 3124-3128 (2008).

6. W. Chang, A. Ankiewicz, J. M. Soto-Crespo, and N. Akhmediev, "Dissipative soliton resonances," Phys. Rev. A 78(2), 023830 (2008).

7. Ph. Grelu, W. Chang, A. Ankiewicz, J. M. Soto-Crespo, and N. Akhmediev, "Dissipative soliton resonance as a guideline for high-energy pulse laser oscillators," J. Opt. Soc. Am. B 27(11), 2336-2341 (2010).

8. E. Ding, P. Grelu, and J. N. Kutz, "Dissipative soliton resonance in a passively mode-locked fiber laser," Opt. Lett. 36(7), 1146-1148 (2011)

9. X. Liu, "Pulse evolution without wave breaking in a strongly dissipative-dispersive laser system," Phys. Rev. A 81(5), 053819 (2010).

10. X. Wu, D. Y. Tang, H. Zhang, and L. M. Zhao, "Dissipative soliton resonance in an all-normal-dispersion erbium-doped fiber laser," Opt. Express 17(7), 5580-5584 (2009).

11. X. Liu, "Coexistence of strong and weak pulses in a fiber laser with largely anomalous dispersion," Opt. Express 19(7), 5874-5887 (2011).

12. X. Zhang, C. Gu, G. Chen, B. Sun, L. Xu, A. Wang, and H. Ming, "Square-wave pulse with ultra-wide tuning range in a passively mode-locked fiber laser," Opt. Lett. 37(8), 1334-1336 (2012).

13. Z. Ch. Luo, W. J. Cao, Z. B. Lin, Z. R. Cai, A. P. Luo, and W. Ch. Xu, "Pulse dynamics of dissipative soliton resonance with large duration-tuning range in a fiber ring laser," Opt. Lett. 37(22), 4777-4779 (2012). 
14. L. Liu, J. H. Liao, Q. Y. Ning, W. Yu, A. P. Luo, S. H. Xu, Z. C. Luo, Z. M. Yang, and W. C. Xu, "Wavebreaking-free pulse in an all-fiber normal-dispersion $\mathrm{Yb}$-doped fiber laser under dissipative soliton resonance condition," Opt. Express 21(22), 27087-27092 (2013).

15. X. Li, Sh. Zhang, H. Zhang, M. Han, F. Wen, and Zh. Yang, "Highly efficient rectangular pulse emission in a mode-locked fiber laser," IEEE Photonics Technol. Lett. 26(20), 2082-2085 (2014).

16. X. Li, S. Zhang, H. Han, M. Han, H. Zhang, L. Zhao, F. Wen, and Z. Yang, "Different polarization dynamic states in a vector Yb-doped fiber laser," Opt. Express 23(8), 10747-10755 (2015).

17. H. Yu, X. Wang, P. Zhou, X. Xu, and J. Chen, "High-Energy Square Pulses in a Mode-Locked Yb-Doped Fiber Laser Operating in DSR Region,” IEEE Photonics Technol. Lett. 27(7), 737-740 (2015).

18. K. Özgören, B. Oktem, S. Yilmaz, F. Ö. Ilday, and K. Eken, " 83 W, 3.1 MHz, square-shaped, 1 ns-pulsed allfiber-integrated laser for micromachining," Opt. Express 19(18), 17647-17652 (2011).

19. S. K. Wang, Q. Y. Ning, A. P. Luo, Z. B. Lin, Z. C. Luo, and W. Ch. Xu, "Dissipative soliton resonance in a passively mode-locked figure-eight fiber laser," Opt. Express 21(2), 2402-2407 (2013).

20. J. H. Yang, Ch. Y. Guo, Sh. Ch. Ruan, D. Q. Ouyang, H. Q. Lin, Y. M. Wu, and R. H. Wen, "Observation of dissipative soliton resonance in a net-normal dispersion figure-of-eight fiber laser," IEEE Photonics J. 5(3), 1500806 (2013).

21. L. Mei, G. Chen, L. Xu, X. Zhang, C. Gu, B. Sun, and A. Wang, "Width and amplitude tunable square-wave pulse in dual-pump passively mode-locked fiber laser," Opt. Lett. 39(11), 3235-3237 (2014).

22. K. Krzempek, "Dissipative soliton resonances in all-fiber Er-Yb double clad figure-8 laser," Opt. Express 23(24), 30651-30656 (2015).

23. N. Zhao, M. Liu, H. Liu, X. W. Zheng, Q. Y. Ning, A. P. Luo, Z. C. Luo, and W. Ch. Xu, "Dual-wavelength rectangular pulse $\mathrm{Yb}$-doped fiber laser using a microfiber-based graphene saturable absorber," Opt. Express 22(9), 10906-10913 (2014).

24. Z. Cheng, H. Li, H. Shi, J. Ren, Q. H. Yang, and P. Wang, "Dissipative soliton resonance and reverse saturable absorption in graphene oxide mode-locked all-normal-dispersion Yb-doped fiber laser," Opt. Express 23(6), 7000-7006 (2015).

25. B. Guo, Y. Yao, Y. Yang, Y. Yuan, L. Jin, B. Yan, and J. Zhang, "Dual-wavelength rectangular pulse erbiumdoped fiber laser based on topological insulator saturable absorber," Photonics Res. 3(3), 94-99 (2015).

26. O. Pottiez, R. Grajales-Coutiño, B. Ibarra-Escamilla, E. A. Kuzin, and J. C. Hernández-García, “Adjustable noise like pulses from a figure-eight fiber laser," Appl. Opt. 50(25), E24-E31 (2011).

\section{Introduction}

Mode-locked fiber lasers represent a unique system capable to generate a variety of pulse shapes. The first mode-locked fiber lasers intended to generate soliton shape pulses $[1,2]$. However the soliton energy is restricted by several tens of picojoules. Therefore, to reach the balance of energy, soliton lasers tend to generate multiple pulses which in most cases are distributed randomly in the cavity. However, even in the first experiments [2-4], it was mentioned that these lasers were capable to generate pulse bunches which circulate in the cavity with fundamental frequency and stable square-shaped pulses with nanoseconds scale duration. It was shown that the pulse duration of square-shaped pulses depends on the pump power, while the amplitude of the pulses maintains nearly constant. The generation of the square-shaped pulses did not obtain a great attention until it was shown that at a given set of laser parameters, the energy of the pulses circulating in the cavity may grow indefinitely [5]. Using a master equation approach for passively mode-locked lasers, the roadmap to increase the pulse energy was proposed. This approach is based on a cubic-quintic Ginzburg-Landau equation (CGLE). The equation contains six coefficients connected with attenuation, bandwidth of a spectral filter, high order Kerr nonlinearity, dispersion, nonlinear amplification, and saturation of nonlinear amplification. Four parameters were fixed while dispersion and nonlinear amplification were varied to find stable solutions. It was numerically shown that the energy of the dissipative solitons of CGLE increases significantly when dispersion and nonlinear amplification are located on a special area in the dispersionnonlinear amplification space. As shown later, the soliton energy can be increased indefinitely for certain values of the system parameters. This phenomenon was called "dissipative soliton resonance" (DSR) (note that an infinite capability for the source of energy is assumed) [6]. The resonance curve in the dispersion-nonlinear amplification space depends strongly on the choice of the remaining system parameters. Later, the set of system parameters at which the DSR exists both for normal and anomalous dispersion was found [7]. In that work it was also found that the pulse shape depends on the dispersion value; and in the normal dispersion 
region, it transforms from Gaussian-like to flat-top shape while the value of the dispersion approaches to the resonance curve. The use of saturable gain, instead of a constant gain, provides a more physically realistic picture of DSR [8]. It was shown that an increment of the saturation energy (i.e. pump power in experiments) results in the transformation of the Gaussian-looking pulse shape to the flat-top shape and then the increment of the pulse duration at constant peak power. The transformation of chirp was also noted. The flat-top pulses were also found using direct calculation of the pulse evolution in an all-fiber ring laser, using nonlinear polarization rotation (NPR) for mode-locking [9]. The results were similar to that obtained in [8]; i.e., an increment of the pulse duration with pump power at nearly constant spectral bandwidth. Most experimental investigations of DSR were done using lasers with the ring configuration using nonlinear polarization rotation for mode-locking [9-17]. However the approach based on the CGLE does not assume a specific laser configuration. Indeed the square-shaped pulses with duration increasing with pump power was demonstrated using figure-of-eight lasers [18-22] and fiber ring lasers using graphene as mode-lockers $[23,24]$. Very recently rectangular pulses were reported in a laser based on topological insulator saturable absorber [25].

Both erbium-doped fiber lasers (EDFL) and ytterbium-doped fiber lasers (YDFL) were developed. The EDFL were investigated with net-normal, net-anomalous, all-anomalous and all-normal cavity dispersion. The ring fiber laser with net-normal cavity dispersion was investigated in [9]. The cavity length was equal to $25.5 \mathrm{~m}$; the average dispersion was equal to $32 \times 10^{-3} \mathrm{ps}^{2} / \mathrm{m}$. The square-shaped pulses reached a temporal width of $250 \mathrm{ps}$ at the maximum available pump power of $550 \mathrm{~mW}$; being the pulse energy of $24 \mathrm{~nJ}$. Its spectrum shown a similar to Gaussian profile, whose bandwidth is slightly decreased from $14 \mathrm{~nm}$ to 11 $\mathrm{nm}$ (FWHM), as the pump power increases. The figure-of-eight laser with net-normal cavity dispersion was presented in [20]. The cavity length was $543 \mathrm{~m}$ with average dispersion of 6.3 $\times 10^{-3} \mathrm{ps}^{2} / \mathrm{m}$. The reported pulse duration reached $350 \mathrm{~ns}$, whereas the pulse energy was of $350 \mathrm{~nJ}$. In both works, the duration of the rectangular pulses increased with increasing pump power, while the peak power remained constant. Similar results were presented in the allnormal ring fiber laser configuration [10]. The cavity length was $165 \mathrm{~m}$ with average dispersion of $4 \times 10^{-3} \mathrm{ps}^{2} / \mathrm{m}$. The maximum pulse duration was $20 \mathrm{~ns}$ while the pulse energy was $280 \mathrm{~nJ}$.

The generation of rectangular pulses was demonstrated also at net-anomalous dispersion in ring-cavity fiber lasers [11,12] and the nonlinear amplifying loop mirror (NALM)-based figure-of-eight fiber laser [19]. The results at all- and net-anomalous dispersions have shown a more complicated dynamics for the laser generation than that at all- and net-normal dispersions. In Ref [11]. a fiber laser with a ring cavity with total length of $720 \mathrm{~m}$ and average dispersion of $-21 \times 10^{-3} \mathrm{ps}^{2} / \mathrm{m}$ was investigated. At low pump powers pulses exhibited the typical spectrum for solitons. However, at pump powers higher than $28 \mathrm{~mW}$, square-shaped pulses coexist with secant hyperbolic-shaped pulses. In Ref [12]. another fiber ring laser was investigated; whose cavity included the 1160-m long highly nonlinear fiber with dispersion of $2.6 \mathrm{ps} / \mathrm{nm} / \mathrm{km}$, a $10-\mathrm{m}$ long SMF-28 fiber with dispersion of $17 \mathrm{ps} / \mathrm{nm} / \mathrm{km}$, and light pulses with duration of $1716 \mathrm{~ns}$ were achieved at pump power of $350 \mathrm{~mW}$. It was noted that the spectrum had two maxima, which is unusual for DSR phenomenon. In addition, the pulses were not exactly squared-shaped, but with a peak power slightly decreasing from the rising to the falling edge, i.e., a trapezoid-shaped pulse. The pulses obtained in Ref [19]. have a visible modulation of the power within each pulse. Moreover, the spectrum shows strong dispersive waves typical for solitons. The pulses with record energy of $2.13 \mu \mathrm{J}$ and pulse duration of 170 ns were reported for the figure-of-eight fiber laser with strong all-anomalous cavity dispersion [22]. The cavity had a length equal to $255 \mathrm{~m}$, and average dispersion of $40 \times 10^{-3} \mathrm{ps}^{2} / \mathrm{m}$. A double clad $\mathrm{Er} / \mathrm{Yb}$ doped fiber was used as the gain medium. The squared-shaped pulses were also obtained using $\mathrm{Yb}$-doped fibers as active medium in fiber ring laser cavities [14-17] and 
figure-of-eight fiber lasers [18,21]. All reported lasers have all-normal dispersion with large average dispersion about $40 \times 10^{-3} \mathrm{ps}^{2} / \mathrm{m}$.

It becomes evident, from the aforementioned, that square-shaped pulses can be obtained in a variety of configurations. It is worth to say also that in these works, the configurations used were similar to those used before to obtain completely different pulse shapes. Therefore, there seems to be a strong correlation between pulse shapes on the one hand, and polarization adjustment/control within the cavity, on the other. In previous works, standard fibers were used in which the polarization state is not well defined and the desired mode of generation was obtained by empirical adjusting of the polarization controllers. Thus, an investigation of the laser with a well-defined polarization state within the cavity is highly desirable. The theoretical investigations show that the dispersion plays an important role. In particular case of DSR phenomenon, the analysis based on CGLE showed that for fixed bandwidth of a spectral filter, high order Kerr nonlinearity, nonlinear amplification, and saturation of nonlinear amplification single square-shaped pulses can be observed in a specific dispersion range. This is one of the fundamental conclusions of the DSR theory. However, to the best of our knowledge, the dependence of the mode of generation on dispersion, while other parameters remains fixed, was never reported. This research is difficult to carry out, particularly, because the operation mode depends also on other parameters, which should have to be fixed. In this work, we present the results of the investigation of the YDFL with all components being polarization maintaining (PM). A semiconductor saturable absorber mirror (SESAM) was used as mode-locker; whereas the average dispersion was varied by appropriate selection of the different fiber lengths within the cavity; including sections of PM photonic crystal fiber (PM PFC) with anomalous dispersion. Thanks to the use of PM elements, all other important parameters remain unchanged when we changed the dispersion of the cavity. We found three different emission regimes, as the absolute value of the netnormal dispersion increases, namely: a square-shaped single pulse, a right-angle trapezoidshaped single pulse, and multiple right-angle trapezoid-shaped pulses, per round-trip.

\section{Experimental setup}

A schematic diagram of the modelocked fiber ring laser is illustrated in Fig. 1. The gain was provided by $1.7 \mathrm{~m}$ of a PM ytterbium-doped, single-clad, optical fiber (PM YDF) [PM-YSFHI by Nufern ${ }^{\circledR}$, core absorption $250 \mathrm{~dB} / \mathrm{m}$ at $975 \mathrm{~nm}$, cut-off wavelength of $860 \pm 70 \mathrm{~nm}$, and numerical aperture of 0.110]. The PM YDF was pumped through a 980/1030 nm PM wavelength division multiplexer (PM WDM) by a $976 \mathrm{~nm}$ pigtailed laser diode, providing a maximum pump power of $600 \mathrm{~mW}$. Next, and following a clockwise direction, the PM YDF was spliced to the $80 \%$ port of an $80 / 20$ PM fiber coupler. The input port of the PM optical coupler was in turn spliced to port 3 of a PM three-port optical circulator (PM OC). Port 2 of the PM OC was spliced to a 12.4-m PM PCF (estimated splice loss $3 \mathrm{~dB}$ ), whereas the other end of the PM PCF was faced to a SESAM (Batop GmbH, high reflection band $1010 \mathrm{~nm} \leq \lambda$ $\leq 1110 \mathrm{~nm}$, relaxation time constant $1 \mathrm{ps}$ ). Finally, the cavity was closed by connecting port 1 of the PM OC to the $1030 \mathrm{~nm}$ port of the PM WDM. The PM PCF (measured attenuation $\leq$ $0.12 \mathrm{~dB} / \mathrm{m}$ ) has anomalous dispersion, while all other fibers, including the pigtails of the elements, have normal dispersion. The PM OC was used not only to force unidirectional operation within the ring cavity, but to provide the possibility of a double pass through the PM PCF, in order to duplicate the value of the anomalous dispersion within the cavity. Light pulses propagates counter-clockwise in this laser; being the output light pulses extracted from the cavity by the $20 \%$ port of the PM optical coupler. Besides the PM PCF and PM YDF, the laser cavity also contains an unavoidable length $(2.5 \mathrm{~m})$ of PM single mode fiber (SMF) due to the pigtails of the several components used. This configuration of the cavity, including this 2.5-m length of PM SMF was our initial configuration. We also investigated the behavior of our laser by adding different lengths of a PM passive fiber (PM980-XP by Nufern, cut-off wavelength of $920 \pm 50 \mathrm{~nm}$, and numerical aperture of 0.120 ) to the original configuration. 
This additional fiber was inserted between port 3 of the PM OC and the PM optical coupler, see Fig. 1. The presence of the additional fiber allows changing the net-dispersion of the cavity. It should be noted that once a satisfactory working condition was found, it was not changed in the subsequent configurations. So, the cavity loss and the SESAM characteristics were the same for all configurations. First, we investigated the initial configuration, and then we increased the total dispersion with the addition of PM passive fiber with lengths of $5.2 \mathrm{~m}$, $7.2 \mathrm{~m}, 8.2 \mathrm{~m}$ and $10 \mathrm{~m}$. We measured by the interferometric technique the dispersion of the different fibers present in the cavity, resulting in $-3.8 \mathrm{ps}^{2} / \mathrm{km}, 24 \mathrm{ps}^{2} / \mathrm{km}$, and $25.2 \mathrm{ps}^{2} / \mathrm{km}$ for the PM PCF, PM SMF, and PM YDF, respectively, at $1030 \mathrm{~nm}$. The total anomalous dispersion was $-0.094 \mathrm{ps}^{2}$ for the PM PCF; while the total normal dispersion was $0.060 \mathrm{ps}^{2}$, and $0.042 \mathrm{ps}^{2}$ for the PM SMF, and PM YDF, respectively. Therefore, without an additional PM SMF, the total cavity dispersion was $0.008 \mathrm{ps}^{2}$, i.e. slightly net-normal dispersion. Note that, because of an inevitable inaccuracy in the length measurements, the dispersion in fact may be slightly anomalous. On the other extreme, with an additional length of $10 \mathrm{~m}$ of PM SMF, the net dispersion of the cavity resulted in $0.262 \mathrm{ps}^{2}$. Finally, the output light pulses were monitored by using a $60 \mathrm{GHz}$ sampling oscilloscope provided with a fast built-in photodetector $(53 \mathrm{GHz}$ ), autocorrelator (maximum scan range $200 \mathrm{ps}$ ), optical spectrum analyzer (wavelength accuracy $\pm 20 \mathrm{pm}$ ), and RF spectrum analyzer (resolution bandwidth 1 $\mathrm{Hz})$.

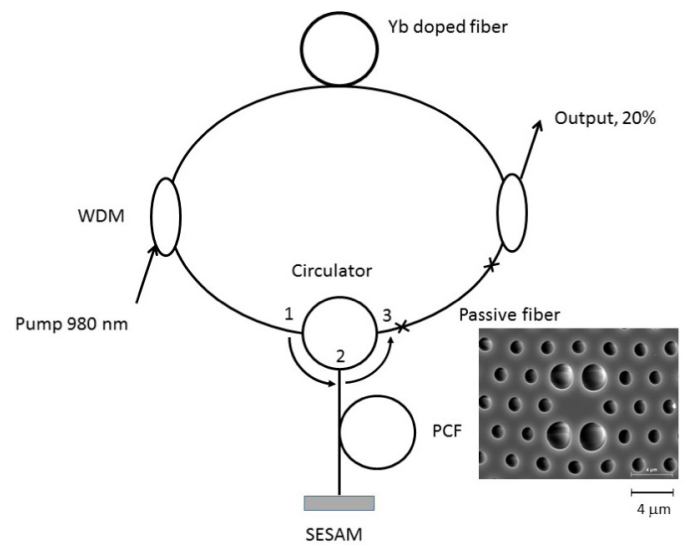

Fig. 1. Experimental setup; inset shows the PM PCF used in the experiments.

\section{Results and discussion}

The laser was self-starting for all configurations. A hysteresis in the mode-locking start-up $(300 \mathrm{~mW})$ and turn-off $(200 \mathrm{~mW})$ was observed, which is otherwise frequently observed in mode-locking lasers. We monitored the pulse waveform, spectrum, an autocorrelation trace, and also characterized the RF performance of the mode-locked pulse train using the RF spectrum analyzer. A signal-to-noise-ratio of more than $40 \mathrm{~dB}$ can be observed for the first fifty harmonics, indicating the high quality of mode-locking, see Fig. 2(a). Figure 2(b) shows the fundamental harmonic, its FWHM is slightly higher than $1 \mathrm{~Hz}$, being ultimately limited by the available resolution bandwidth of our RF analyzer $(1 \mathrm{~Hz})$.

As a function of the pump power, and without additional passive fiber the pulses have the waveforms shown in Fig. 3(a). For this configuration, the net-normal dispersion of the cavity was $0.008 \mathrm{ps}^{2}$, the cavity length was $29 \mathrm{~m}$, and pulse repetition frequency of $7.04 \mathrm{MHz}$. We found that the pulse width increased with the pump power, while the pulse intensity reduces very slightly. The dependence of the pulse duration on the pump power is presented in Fig. 3 (b). Furthermore, we can see that the temporal intensity profile of the light pulses transforms from Gaussian-like to square-shaped as the pump power increases. This transition is far from 
being gradual, Gaussian-like waveforms were only observed in a narrow margin of pump powers, once the start-up threshold was surpassed. On the other hand, each square-shaped pulse presents a nearly constant intensity, i.e. flat-top. The rise time of the square-shaped pulses, measured with the oscilloscope, was equal to $16 \mathrm{ps}$; whereas the fall time was of 27 ps.
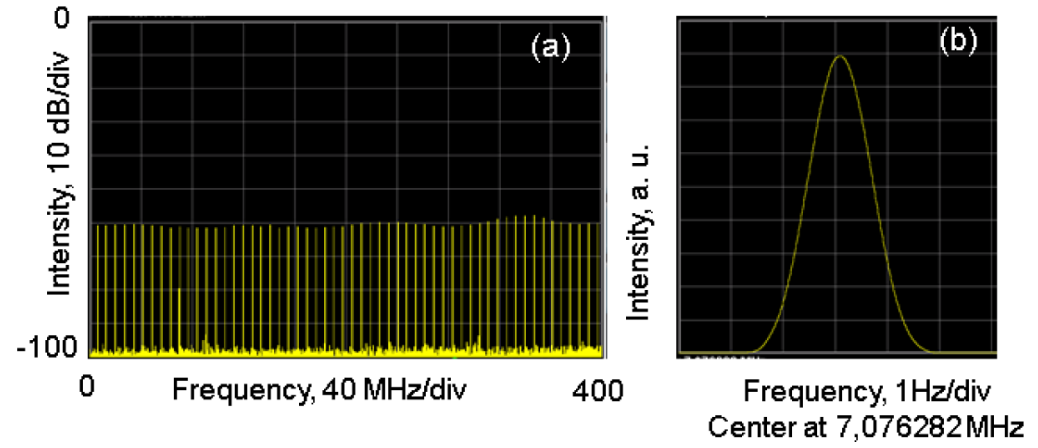

Fig. 2. (a) RF spectrum of the light pulses for the initial configuration. (b) Fundamental RF peak at the highest available resolution.
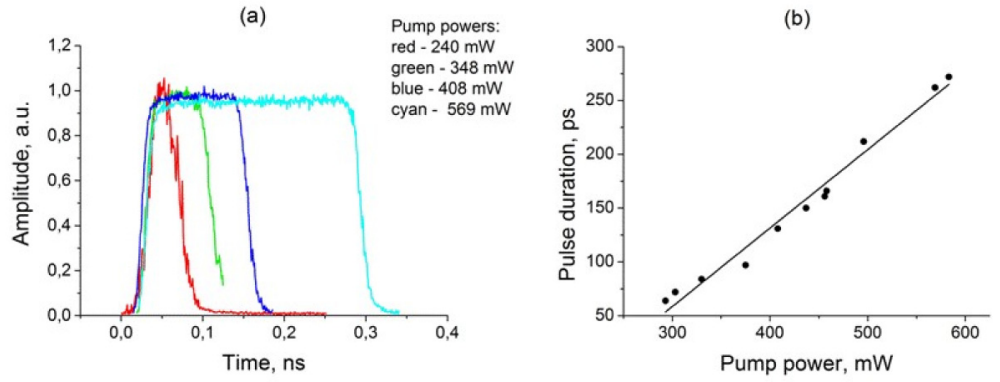

Fig. 3. (a) Oscilloscope traces of pulses for the initial configuration. (b) Pulse duration vs. pump power; the solid line presents the linear fit.

Figure 4 presents the dependence of the average power on the pulse duration together with its corresponding fitting by a linear function. Taking into account average power, pulse duration, and frequency repetition rate we can estimate the peak power of pulses as $170 \mathrm{~mW}$. The moderately low value of average power can be explained by a combination of multiple factors, between them: the high splice loss between the PCF and one of the fiber optic coupler ports, a moderately low recoupling of light reflected from the SESAM faced to one cleaved facet of the PCF, and the use of an 80/20 optical coupler. We estimate the total cavity losses would be between $10 \mathrm{~dB}$ and $13 \mathrm{~dB}$.

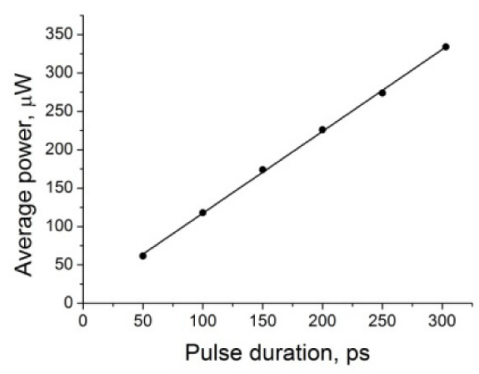

Fig. 4. The dependence of the output average power on the pulse duration. 
The spectral shapes of the pulses were very similar at all pump powers. Figure 4(a) shows an example of the spectrum measured at the pump power equal to $575 \mathrm{~mW}$, together with its corresponding Gaussian fit. On the other hand, an attempt to fit the same experimental data with a secant hyperbolic profile, results in a higher deviation. The bandwidth decreases slightly with pump power, see Fig. 5(b). It is worth to say, that similar spectrum shapes and bandwidth dependence with pump power were calculated and measured in [9].

(a)

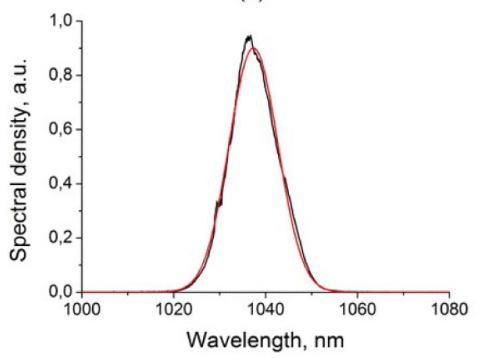

(b)

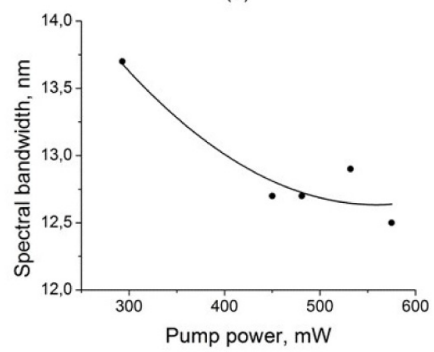

Fig. 5. (a) Experimentally measured spectrum of the light pulse (black line) together with its corresponding Gaussian fit (red line). (b) Dependence of the spectral bandwidth on the pump power: solid dots - experimental data, line - the polynomial fit of the second order.

The square shaped envelope can be observed also for noise like pulses [26]. Noise-like pulses have a very complex internal structure and consist of a very big number of ultra-short pulses within the envelope; whereas in DSR, there is a single light pulse, whose amplitude can be more or less noisy. Although in both cases the oscilloscope traces can be very similar, the autocorrelation traces are significantly different. Next, we performed an intensity autocorrelation measurement. According to the autocorrelation theory, a given square-shaped pulse of pulse width $\Delta t$ has an isosceles triangle for intensity autocorrelation, whose FWHM is also $\Delta t$. Figure 6 shows a typical example of the measured autocorrelation trace for a square shaped pulse of 68 ps (FWHM). The autocorrelation trace has the expected isosceles triangle shape, with a FWHM of $\sim 70$ ps. The difference between measured value could be due to the high noise content in the autocorrelation trace, which in turn is a consequence of a moderately low peak power-average power product of the signal measured $\left(2 \times 10^{-5} \mathrm{~W}^{2}\right)$. However, this value is higher enough to realize a reliable measurement. On the other hand, it is important to note the absence of a short high peak in the center of the autocorrelation trace, which is typical for noise-like pulses.

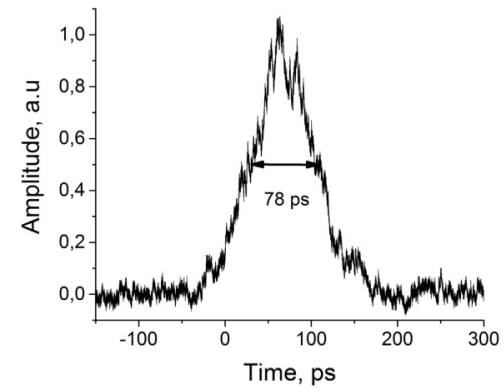

Fig. 6. Autocorrelation trace for a 68 ps pulse width square-shaped DSR light pulse.

The insertion of the $5.2 \mathrm{~m}$ long PM SMF increments the dispersion of the cavity from $0.008 \mathrm{ps}^{2}$ up to $0.139 \mathrm{ps}^{2}$. The profile of the light pulses for this dispersion remains squareshaped, see Fig. 7(a), however the pulse duration increased significantly for the same pump powers. It is worth to say that each square-shaped pulse remains of nearly constant intensity, i.e. flat-top, as in the preceding case. 

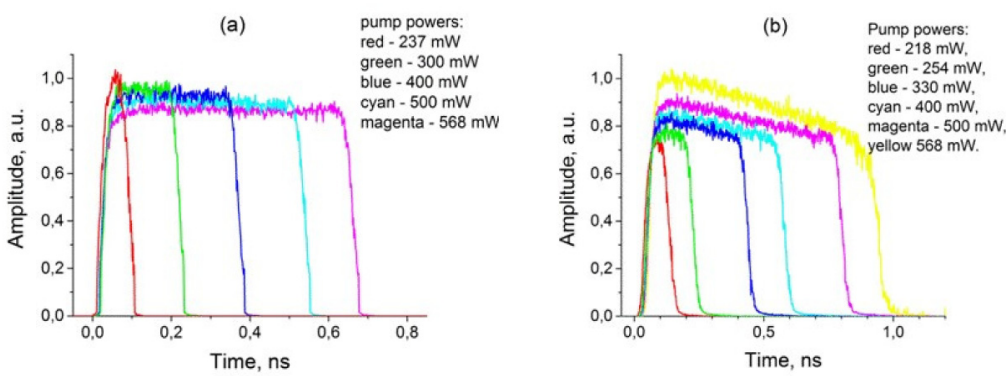

Fig. 7. Oscilloscope traces of pulses for the configuration with $5.2-\mathrm{m}$ of passive fiber inserted to the cavity (a) and with 7.2-m (b).

Next, we added a 7.2-m long PM SMF to the initial configuration; thus, the net dispersion grows to $0.187 \mathrm{ps}^{2}$. In this case, there is a noticeable change in the temporal profile of the output light pulse, from the original square-shaped to trapezium-shaped, see Fig. 7(b). Now, each single light pulse no longer shows a nearly constant intensity as a function of time. We can see also that the peak power grows slightly with the pump power. With the $8.2 \mathrm{~m}$ long passive fiber inserted in the cavity (cavity net dispersion $0.210 \mathrm{ps}^{2}$ ), the pulse shape substantially remains the same, as with the $7.2 \mathrm{~m}$ long PM SMF. The pulses again show the preceding trend, i.e., increasing their duration as the pump power increases. When $10-\mathrm{m}$ length PM SMF was added, the net dispersion grows to $0.256 \mathrm{ps}^{2}$. Again, a single trapeziumshaped light pulse by round-trip can be observed, however only in a restricted range of pump power, between $216 \mathrm{~mW}$ and $306 \mathrm{~mW}$. When the pump power is increased further, the single pulse splits in two equidistant trapezium-shaped light pulses by round-trip, which again show the preceding trend, i.e., increasing their width as the pump power increases.

Figure 8 shows the dependencies of the pulse duration on the pump power for all the investigated configurations. There is a linear trend in each studied case, which is otherwise expected for DSR. The interesting fact is that the slopes of the dependencies grow with the increment of the net-normal dispersion; being $0.73 \mathrm{ps} / \mathrm{mW}, 1.68 \mathrm{ps} / \mathrm{mW}, 2.22 \mathrm{ps} / \mathrm{mW}, 2.65$ $\mathrm{ps} / \mathrm{mW}$, and $4.36 \mathrm{ps} / \mathrm{mW}$ for PM SMF lengths equal to 0 (initial configuration), $5.2 \mathrm{~m}, 7.2 \mathrm{~m}$, $8.2 \mathrm{~m}$, and $10 \mathrm{~m}$, respectively. When 5.2-m, 7.2-m, and 8.2-m length of PM SMF was inserted in the cavity, only one pulse circulated in the cavity at all range of available pump power. However, when the $10 \mathrm{~m}$ long PM SMF was inserted in the cavity, a single pulse by round-trip was observed only if the pump power was lower than $310 \mathrm{~mW}$. When the pump power is higher, the pulse splits in two trapezium-shaped pulses by round-trip. However, even in this case, for pump powers lower than $310 \mathrm{~mW}$, this trend of pulse width increment with dispersion remains.

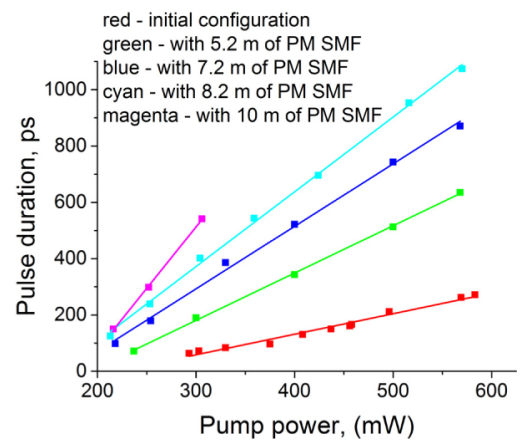

Fig. 8. Experimentally measured dependencies of the pulse duration on the pump power together with their linear fits. 


\section{Conclusion}

A full PM ring fiber laser using SESAM as mode-locker with four different values of netnormal dispersion in the range from $0.008 \mathrm{ps}^{2}$ to $0.262 \mathrm{ps}^{2}$ was investigated. In order to maintain the total dispersion low at $\sim 1030 \mathrm{~nm}$, a section of a PM PCF was included within the cavity, with a net anomalous dispersion. The use of PM elements in the cavity allows the change of the cavity dispersion, without altering other important parameters, which establish the regime of laser operation. We found three different regimes, depending on the cavity dispersion, at dispersions below $0.139 \mathrm{ps}^{2}$, DSR was observed. The square-shaped light pulses have the typical for DSR linear dependence on the pump power, independent on pump power amplitude, and Gaussian like spectrum. At dispersion of $0.187 \mathrm{ps}^{2}$ and $0.210 \mathrm{ps}^{2}$, a single trapezium-shaped light pulse in the cavity was observed. The pulse duration varied approximately linearly with pump power, as for DSR; however its amplitude was not constant and increased with pump power. At dispersion of $0.256 \mathrm{ps}^{2}$, two trapezium-shaped light pulses were observed in the cavity per round trip, if the pump power exceeded $306 \mathrm{~mW}$.

\section{Acknowledgment}

Financial support from the Ministerio de Economía y Competitividad of Spain and FEDER funds (project TEC2013-46643-C2-1-R), and the Generalitat Valenciana (project PROMETEOII/2014/072) is acknowledged. C. Cuadrado-Laborde acknowledges financial support from the Universidad de Valencia (Ref.: UV-INV-EPC14-186393) and CONICET (project PIP 11220150100607CO). E. A. Kuzin was supported by CONACYT project 130966. 